\title{
Adaptación y validación del Cuestionario de Relación Docente en el Nivel Superior*
}

\section{Adaptation and validation of the Questionnaire on Teacher Interaction in Higher Education}

\author{
Julieta Laudadío** y Claudia Mazzitelli***
}

\begin{abstract}
* Investigación realizada en el marco de una Beca Posdoctoral otorgada a la primera autora, por el
Consejo Nacional de Investigaciones Científicas y Técnicas (CONICET).

***Doctora en Educación. Becaria Posdoctoral del Consejo Nacional de Investigaciones Científicas y

Técnicas (CONICET). E-Mail: julietalaudadio@gmail.com

***Doctora en Educación y Profesora de Nivel Medio y Superior de Física. Profesora Titular de cátedras de Formación Docente. Miembro de la Carrera del Investigador Científico del Consejo

Nacional de Investigaciones Científicas y Técnicas (CONICET), y Directora e Investigadora del Instituto de Investigaciones en Educación en las Ciencias Experimentales de la Universidad

Nacional de San Juan. E-Mail: mazzitel@ffha.unsj.edu.ar
\end{abstract}

Universidad Nacional de San Juan.

Consejo Nacional de Investigaciones Científicas y Técnicas (CONICET).

Argentina

\section{Resumen}

La docencia exige una estrecha relación entre el profesor y los estudiantes que requiere para su perfeccionamiento una adecuada explicitación y reflexión por parte de los docentes. Algunas investigaciones señalan la importancia que la forma de relacionarse tiene en el desarrollo de los alumnos y sus logros.

El objetivo del trabajo que se informa fue evaluar la validez y fiabilidad del Cuestionario sobre la Relación Docente (QTI, Wubbels, Brekelmans \& Hooymayers, 1991), adaptado al ámbito universitario por Soerjaningsih, Fraser y Aldridge.

Dicho instrumento está compuesto por 48 ítems y permite identificar la tipología de comportamiento predominante en el docente a partir de dos dimensiones: proximidad (cooperación - oposición) e influencia (dominación - sumisión). El cuestionario fue administrado a 256 estudiantes de primero y segundo año de carreras relacionadas con las Ciencias Naturales y las
Ciencias de la Salud de universidades privadas y públicas de la Provincia de San Juan (República Argentina). Para evaluar la fiabilidad se aplicó el Alpha de Cronbach y se realizó un análisis factorial para estudiar la validez de constructo. Los resultados indican la existencia de una estructura bidimensional: el factor 1 está compuesto por ítems que evalúan la proximidad en la relación docente e incluye ítems positivos que corresponden a la subdimensión cooperación e ítems negativos que corresponden a la subdimensión oposición. El factor 2 está compuesto por ítems que evalúan la influencia con respecto a la dimensión dominación. Se obtuvo para el factor 1, un Alpha de Cronbach igual a .92 para cooperación y un Alpha de Cronbach igual a .84 para oposición. En el factor 2, el Alpha es igual a .61. El autoinforme muestra globalmente un nivel de fiabilidad aceptable.

Palabras clave: Educación superior; Relación docente; Estudiantes; Validez; Fiabilidad. 


\begin{abstract}
As regards the education action, teaching is not only a merely technical activity, or an action without communication, bonds or social contact. On the contrary, it requires a close relationship between the teacher and the students, which -in order to be effectively developed- needs the teachers to be adequately conscious and reflective about the aims they want to achieve through the communicative action. During the last decades, relationships between teachers and students have become a central focus for theories and investigation, and many aproaches show the importance that the relationship has as regards the students' development and their accomplishments (Cohen \& Amidon, 2004; Páramo, 2008). Indeed, it has been stated that the best education is a product of the best relationship or pedagogical bond.
\end{abstract}

Teaching demands a close relationship between the teacher and the students, which requires an adequate explicitness and reflection on the part of the teacher for its effective development. Some research highlights the importance this relationship has for the students' development and achievements. This work aims at evaluating the validity and reliability of the Questionnaire on Teacher Interaction (QTI) applied in higher education by Soerjaningsih, Fraser and Aldridge. This instrument includes 48 items and enables the identification of the teacher's predominant behavior according to two dimensions: proximity (cooperation-opposition) and influence (domination-submission). The questionnaire was applied to 256 students attending the first two years of courses of study related to Natural Science and Health Science at both public and private universities in the province of San Juan (Argentina). To evaluate the reliability, the Cronbach Alpha was applied, and the validity of the construct was studied by making a factorial analysis. The results indicate the existence of a two-dimensional structure: factor 1 is constituted by items that evaluate the proximity of the student-teacher relationship; it includes positive items that correspond to the cooperation subdimension and negative items that correspond to the opposition subdimension. Factor 2 is constituted by items that evaluate the influence in relation to domination. As regards reliability, when studying Factor 1, a Cronbach Alpha of .92 was obtained for cooperation and a Cronbach Alpha of .84 for opposition. Factor 2 had an Alpha of .61. The selfreport globally shows an acceptable level of reliability.

Summarising, favorable evidence was obtained about the discrimination of the items: factorial validity and the instrument's reliability. These results are important to understand the dynamics of the processes implied in the student-teacher relationship. Taking into account these results, it is considered that the QTI can be used as a guide to improve the interpersonal relationships and to help teachers in their professional development. Using this instrument can be a valuable tool, both for investigation and intervention and prevention programs. Good teachers should be able of empathizing with students, listening to them better and understanding their world. In this sense, there should be training plans for teachers to be conscious of the relational dimension of their education action and for them to receive the necessary courses to be able to optimize the great educational force that their practice has on students' development. Indeed, incorporating strategies to develop and strengthen relational capabilities in teachers during the teachers' training course will contribute to students, interpersonal relationships which will favor the academic performance, and it will enable teachers' accomplishments and professional success.

Key words: Higher education; Teacher interaction; Students; Validity; Reliability.

\section{Introducción}

La docencia, como acción educadora, no se reduce a una actividad meramente técnica, ni a una acción desprovista de comunicación, vínculo y contacto social. Por el contrario, exige una estrecha relación entre el profesor y los estudiantes, que requiere para su efectivo desarrollo la adecuada conciencia y reflexión de parte de los docentes acerca de los objetivos que se pretenden al- 
canzar a través de la acción comunicativa. Al respecto, Velasco y Alonso de González (2008) sostienen que si se suprime el diálogo no hay educación posible. Al mismo tiempo señalan que saber acerca de algo no garantiza que se lo ponga en práctica, lo cual refleja la situación actual del educador que sabe acerca del diálogo, pero en muchas ocasiones no logra concretarlo en su tarea en el aula. Recientes investigaciones (Carbonero, Martín-Antón \& Reoyo, 2011; Jong et al., 2014; Klein, 2011; Veldman, van Tartwijk, Brekelmans \& Wubbels, 2013) señalan la importancia de este aspecto en la formación inicial, ya que el aprendizaje para ser docente posee un carácter relacional pues tiene que ver con las relaciones personales que se establecen con los demás actores educativos: directivos, profesores y específicamente los alumnos.

En los últimos dos decenios, las relaciones entre docentes y estudiantes se han convertido en un foco central de la teoría y la investigación, y muchas manifiestan la importancia que la forma de relacionarse tiene en el desarrollo de los alumnos y sus logros (Cohen \& Amidon, 2004; Páramo, 2008). En efecto, se ha afirmado que la mejor educación es fruto de la mejor relación o vínculo pedagógico. En el marco de una perspectiva interpersonal de la enseñanza se encuentran investigaciones que revelan una influencia positiva de la relación docente sobre los resultados cognitivos y afectivos de los estudiantes (Wubbels \& Brekelmans, 2005). Al respecto, estudios recientes (LaBillois \& Lagacé-Séguin, 2009; Zapata, Rojas, \& Gómez, 2010) señalan también la necesidad de profundizar en los factores intervinientes en la relación de confianza que generan los profesores en sus estudiantes.

El estudio de las relaciones e interacciones que se establecen entre profesor y alumnos en el contexto del aula cuenta con una dilatada trayectoria en la investigación educativa y psicoeducativa. Una y otra vez los investigadores han dirigido su atención al estudio de estas relaciones e interacciones con los más diversos objetivos: identificar los rasgos del profesor ideal (Darling-Ham- mond, 2008; Salazar, 2006), describir los estilos de enseñanza (Gargallo López, 2008; Gargallo López, Sánchez Peris, Ros Ros \& Ferreras Remesal, 2010), analizar el clima socioemocional de la clase (Carbonero et al., 2011; LaBillois \& Lagacé-Séguin, 2009), describir los comportamientos del profesor eficaz (Rocha Chávez, 2013; Zhang, 2011), etc. Asimismo manifiestan que de la preocupación por identificar las características de los docentes eficaces, se ha pasado al interés por determinar los métodos de enseñanza eficaces y de aquí al propósito de identificar con precisión las competencias profesionales de los docentes eficaces con el fin de situarlas en el núcleo de las actividades de formación del profesorado. El cambio importante se produce en el tránsito desde la consideración del contexto del aula, a una visión del aula como contexto de enseñanza y aprendizaje. Lo cual explicaría el auge del estudio de las prácticas educativas y del resurgimiento del interés por el análisis de la interacción docente (Coll \& Sánchez, 2008).

La enseñanza en sí misma requiere un repertorio de competencias interpersonales que sea a la vez amplio y flexible. Los profesores deben desarrollar las habilidades comunicativas que les permitan establecer relaciones favorables con los alumnos (Bibi, Kazmi, Chaudhry \& Khan, 2015; Brackett, Palomera, Mojsa-Kaja, Reyes \& Salovey, 2010; Wubbels \& Brekelmans, 2005). La construcción de relaciones es una condición previa para lograr un clima positivo en el aula. Sin este aspecto, los docentes no pueden desarrollarse plenamente en su práctica, de allí la importancia de prestar mayor atención a las habilidades interpersonales en el desarrollo profesional docente (Fernández Berrocal \& Ruiz Aranda, 2008; Palomera Martín, Gil-Olarte Márquez, \& Brackett, 2006; Palomera Martín, Fernández Berrocal, \& Brackett, 2008).

\section{Vínculo pedagógico}

Wubbels y Levy (1997) han señalado luego de 15 años de investigaciones la im- 
portancia de que los profesores desarrollen habilidades interpersonales para crear un clima en el aula que favorezca el aprendizaje. A su vez, manifiestan la necesidad de contar con instrumentos que permitan valorar la capacidad de los profesores para comunicarse con los alumnos y las cualidades de dicha relación. La revisión de la literatura muestra que una adecuada relación profesor-alumno se caracterizan por un alto grado de influencia del docente y proximidad a los estudiantes. En este sentido se puede decir que es el docente quien crea ambientes propicios, da orden, motiva, ajusta sus dinámicas a las características del grupo y potencializa las destrezas de sus alumnos. De allí que la clave para conseguir una educación de calidad es contar con docentes bien preparados.

Tal como lo demuestran diversas investigaciones (Vaillant, 2005, 2007; Zabalza, 2003), la calidad del desempeño de los docentes, entre otros factores, tiene una alta incidencia en el logro de aprendizajes. Vidal (2002) afirma que la calidad docente no es exclusiva responsabilidad del profesor, sino que también es una responsabilidad de los sistemas y políticas educativas en su conjunto. Es por ello que se puede afirmar que el nivel de desempeño docente abarca dos aspectos: uno interno, con un grado de responsabilidad centrada principalmente en el docente y otro externo, con un importante grado de responsabilidad de las instituciones, las políticas de formación y evaluación educativa. La dimensión interna se refiere a la disposición que cada profesor tiene frente a su desempeño docente. Se relaciona con las metas que cada uno se plantea en el aspecto personal y profesional, es decir con el ideal que desearía alcanzar en el ejercicio de su profesión. Mientras que la dimensión externa se relaciona con los aspectos que en la práctica facilitan o dificultan el desarrollo y manifestación de niveles de excelencia docente. Se refiere a las oportunidades que el sistema y la organización educativa ofrecen para el desarrollo del trabajo del profesor en condiciones de excelencia (Vidal, 2002). El estudio realizado estuvo centrado en la dimensión interna, es decir en las disposiciones del personales del docente.

Al respecto Villa Sánchez (2008) afirma que el papel del docente universitario se ha hecho mucho más complejo debido a los distintos roles que debe desempeñar ya que su tarea no está orientada sólo la mera enseñanza, sino también a favorecer el aprendizaje autónomo de los estudiantes. Lo cual supone nuevas competencias ya que exige a su vez asumir un enfoque que conlleva un comportamiento docente más rico y complejo. A esto se suma que en la actualidad los profesores, desafortunadamente, experimentan con más frecuencia situaciones estresantes y emociones negativas en las aulas. Las exigencias del entorno laboral pueden producir tensión o ansiedad, que mantenidas en el tiempo ocasionan el llamado síndrome de estar quemado en el trabajo o burnout (Pena \& Extremera, 2012) y es una realidad que preocupa tanto a instituciones como a los sindicatos y responsables de políticas educativas. Dichas emociones, como por ejemplo la ansiedad, interfieren en la capacidad cognitiva (Choi, Vickers \& Tassone, 2014), mientras que las positivas aumentan la capacidad creativa para generar nuevas ideas (Mortan, Ripoll, Carvalho \& Bernal, 2014) y por tanto la capacidad de enfrentar las dificultades (Bibi et al., 2015). Así, la experiencia satisfactoria de los docentes puede aumentar su bienestar y también el de los alumnos. Este efecto positivo además puede generar en la clase un clima favorable para el aprendizaje (Páez Cala \& Castaño Castrillón, 2015) .

En este sentido se puede afirmar que la práctica educativa es un sistema interactivo en el que inevitablemente las emociones tienen un rol fundamental. Las emociones y las habilidades relacionadas con su manejo, afectan a los procesos de aprendizaje, a la salud mental y física (Fernández-Abascal \& Martín-Díaz, 2015; Holinka, 2015), a la calidad de las relaciones sociales (Di Fabio, 2015) y al rendimiento académico y laboral (Brackett et al., 2010). Algunos autores consideran la docencia como una de las profe- 
siones más estresantes, sobre todo porque implica un trabajo diario basado en interacciones sociales en las que el docente debe hacer un gran esfuerzo para regular no sólo sus propias emociones sino también las de los estudiantes (Palomera, Fernández Berrocal \& Brackett, 2008).

De allí la conveniencia de conocer cuáles son las competencias emocionales que los docentes deben potenciar en la práctica educativa, tanto en lo que respecta a las influencias directas como indirectas, y detectar cuáles son las necesidades formativas del profesorado al respecto. Asimismo algunos investigadores (Abarca, Marzo \& Sala, 2002) señalan la importancia del desarrollo de capacidades tales como: el conocimiento y la comunicación de las emociones, la aceptación de uno mismo, el autocontrol, la empatía, la capacidad de resolver problemas, la habilidad para establecer vínculos, la habilidad para automotivarse y la asertividad.

De este modo queda claro que en la interacción profesor-alumno, el profesor tiene un importante papel y su buen desempeño depende de la habilidad que tenga para establecer una comunicación adecuada con sus estudiantes. En esta línea, investigaciones en el Nivel Superior (Paes da Silva \& Kalil de Freitas Castro, 2003) indagan acerca de los aspectos de la comunicación verbal y no verbal del docente que interfieren y que facilitan la interacción educativa. Entre ellos cabe destacar como aspectos facilitadores: las características del profesor postura actitudinal y física, aparte del conocimiento del profesor-, las características del alumno -tanto individuales como grupales-, regularidad de contacto y características comunes a los profesores y alumnos. Por otra parte, identifican como aspectos obstaculizadores de la comunicación del docente: lo verbal -tono y ritmo de la vozy lo no verbal como los gestos, expresiones corporales, las expresiones del rostro, la postura, actitudes, etc.

Otros estudios señalan el impacto de la disposición emocional, tanto de docentes como de estudiantes, en el proceso de en- señanza y aprendizaje. Al respecto Delgado Arriagada (2004) manifiesta que la toma de conciencia de profesores y estudiantes sobre el papel de las relaciones que establecen encuentran un espacio propicio para promover un aprendizaje de calidad. Dicho aprendizaje es analizado, desde la perspectiva de los estudiantes, a partir de cuatro categorías: contenidos de la asignatura y cumplimiento de sus objetivos; metodologías empleadas por el profesor y participación del estudiante; relación con el profesor y precepción del estudiante y finalmente la relación del estudiante con sus compañeros. Dicho estudio sostiene que todo profesor que ha tomado conciencia de sus competencias para provocar cambios que mejoren el aprendizaje de sus estudiantes aporta calidad a la docencia, ya que es el profesor quien puede guiar los estilos de interacciones propicias para el aprendizaje como clases dinámicas, clima grato, espacios de participación y opinión de los estudiantes, etc. Además le corresponde mediar el rumbo de aquellas estrategias que generen situaciones invalidantes para promover el aprendizajes significativos, por ejemplo, cuando el estudiante percibe que el profesor impone su opinión, no acepta otros puntos de vistas, es poco claro y la clase es rígida y reiterativa.

Asimismo, Wubbels y Brekelmans (2005) proponen un modelo para describir la relación docente-estudiante en términos del comportamiento de los profesores. Es decir, que los estudios en esta línea se centran en las percepciones de los estudiantes de lo que ocurre en el aula, lo que piensan acerca de su profesor y lo que aprenden. En este sentido, partiendo de la descripción de la relación docente-estudiante, examinan el comportamiento no verbal y la posición espacial del profesor en la clase, las diferencias de percepción en las relaciones de los profesores con las diferentes clases o con diferentes estudiantes en una misma clase y, finalmente, las intervenciones para mejorar las relaciones de clase.

Las percepciones de los estudiantes sobre sus relaciones con sus profesores han sido estudiados por estos autores a partir del 
Model for Interpersonal Teacher Behavior (MITB) desarrollado por Timothy Leary en 1957. El MITB está conformado por dos dimensiones: influencia (dominio-sumisión) y proximidad (oposición-cooperación). Estas dimensiones proporcionan una visión general de los comportamientos típicos de los docentes y pueden ser representados en un sistema de coordenadas ortogonales. Las dos dimensiones, representadas como dos ejes, son la base para definir ocho perfiles de comportamiento de los profesores. La dimensión proximidad pone el foco en los componentes afectivos y se refiere a las conductas de apoyo o de expresión de emociones del profesor hacia el alumnado. En un extremo se sitúan los comportamientos de amabilidad, ayuda y comprensión del docente y en el otro extremo los que muestran insatisfacción y represión de las conductas de los estudiantes.

La dimensión influencia está más centrada en la enseñanza, en quien controla, dirige o gestiona el proceso de comunicación, en las conductas del profesor dirigidas a proporcionar guías y estructura sobre la enseñanza y el comportamiento. En un extremo encontramos el docente que domina o ejerce el control, bien liderando con claridad la enseñanza y el aula, bien dando libertad o autonomía a los estudiantes para que decidan lo que pueden hacer o cómo hacerlo; en el otro extremo, el docente no ejerce esa influencia positiva, bien porque sobredimensiona su control imponiéndose al alumnado, bien porque se muestra inseguro o indeciso (Wubbels \& Brekelmans, 2005).

La perspectiva interpersonal (Wubbels \& Brekelmans, 2005) hace especial hincapié en que los estudiantes están expuestos y se implican en muchos procesos relacionales simultáneos, es decir, que la relación alumno-profesor implica un proceso de interacción dinámico en el aula, en donde la eficacia de la tarea docente se mide en términos de los patrones recurrentes y relativamente estables que han surgido a partir de las interacciones que tienen lugar cada día en el aula (den Brok, Brekelmans \& Wubbels, 2006).
Investigaciones recientes en esta línea (Wubbels et al., 2012) señalan que un problema central en el estudio de las relaciones interpersonales en educación es la cuestión de la causalidad frente a la reciprocidad. Desde una perspectiva teórica, los avances en la comprensión de las relaciones profesor-alumno requieren la consideración adicional de los caminos de influencia y causalidad. El profesor, con su conducta emocionalmente competente y su estilo de enseñanza, se convierte en facilitador o inhibidor del aprendizaje y motivación de los alumnos. Puede estimularlos a asumir la responsabilidad por sus éxitos y fracasos. Asimismo, cuando los alumnos perciben este apoyo socio-afectivo (preocupación de sus docentes), se motivan, comprometen e implican en las actividades académicas de la clase, se esfuerzan y asumen con responsabilidad las metas académicas (Ivcevic \& Brackett, 2014; Látalová \& Pilárik, 2015; Limonero et al., 2015; Wentzel, 1997). Otros de los aspectos que señalan los investigadores es la necesidad de contar con instrumentos y herramientas de análisis que podrían contribuir a desentrañar los vínculos entre las relaciones interpersonales y otras variables (Mainhard, Brekelmans \& Wubbels, 2011).

Los antecedentes en este campo de estudio coinciden en que son cada vez más quienes sostienen que hay una relación bidireccional y de interdependencia en el análisis de las interacciones del docente con sus estudiantes (Moreno García, 2010), teniendo en cuenta que las relaciones saludables en un aula son un prerrequisito para implicar a los estudiantes en las actividades de aprendizaje (Wubbels \& Brekelmans, 2005). De esta manera se comprende la importancia del estudio de la percepción que tienen los estudiantes del comportamiento de su profesor, estando fuertemente relacionada con el rendimiento y la motivación de los estudiantes en las distintas áreas de aprendizaje (den Brok, Brekelmans, \& Wubbels, 2004).

\section{Evaluación del vínculo pedagógico}

Atendiendo a la variedad de perspectivas 
teóricas no es de extrañar que encontremos diversidad de instrumentos que se han empleado para investigar la capacidad de relacionarse tanto de los adultos (Mikulic, Crespi \& Radusky, 2015) como de los niños (Oros \& Fontana Nalesso, 2015) y adolescentes (Samper-García, Mesurado, Richaud \& Llorca, 2016). Las herramientas van desde cuestionarios que han sido desarrollados específicamente para medir las cualidades interpersonales de los docentes, a extractos de los instrumentos más amplios existentes con el mismo propósito. Aunque el uso de los instrumentos existentes limita el número y el tipo de variables que pueden ser investigadas, presenta una clara ventaja de recursos para el desarrollo de nuevas medidas ya que los datos pueden ser recogidos o reutilizados de manera más eficiente.

La siguiente es una breve descripción de los instrumentos que fueron específicamente desarrollados para medir las relaciones entre profesores y alumnos. A partir de la revisión de los antecedentes empíricos se destacan los siguientes instrumentos:

1.- Student Perception Inventory (Rayneri, Gerber \& Wiley, 2006) o Inventario de Percepción del Alumno: El cuestionario consta de 30 ítems sobre las percepciones de los estudiantes en sus ambientes de clase.

2.- Teacher Communication Behavior Questionnaire (She \& Fisher, 2000): Cuestionario Comportamiento Comunicacional del Docente. Evalúa la percepción de los alumnos de la conducta de comunicación docente a través de 40 ítems.

3.- What Is Happening In This Classroom? (Aldridge, Laugksch, Seopa \& Fraser, 2006): ¿Qué está pasando en esta clase? Cuestionario de 60 ítems acerca de las percepciones del ambiente de la clase y preferencias de los estudiantes.

4.- Student-Teacher Relationship Scale (Saft \& Pianta, 2001): mide la relación docente a partir de tres dimensiones: cercanía, conflicto y dependencia. El cuestionario consta de 28 ítems. La cercanía hace referencia al grado de afecto, calidez y la comunicación abierta; el conflicto describe el grado de negatividad y discordancia y finalmente la dependencia evalúa el grado de unión y posesividad en la interacción docente.

5.- Questionnaire on Teacher Interaction (Wubbels, Brekelmans \& Hooymayers, 1991) o Cuestionario sobre Interacción Docente: El cuestionario consta de 48 ítems sobre los diversos aspectos de las interacciones docentes donde se examinan 8 dimensiones del comportamiento.

Para esta investigación se considera más apropiado este último cuestionario, ya que los indicadores que define para identificar las cualidades de la relación docente se corresponden con el modelo propuesto en este estudio. Asimismo es uno de los instrumentos más utilizado en investigaciones tanto para el nivel de enseñanza básica (den Brok, Fishe, \& Scott, 2005), como secundaria (Lapointe, Legault \& Batiste, 2005) y en el ámbito universitario (den Brok et al., 2004). También es aplicado en el ámbito de la formación de profesores principiantes (Nijveldt, Beijaard, Brekelmans, Verloop \& Wubbels, 2005) y en el de la enseñanza de las ciencias (Khine \& Fisher, 2004; Telli, den Brok \& Cakiroglu, 2010).

El Cuestionario sobre la Relación Docente (QTI) sigue la tradición en la investigación de entornos de aprendizaje través de las percepciones de los estudiantes en el aula. Aunque este programa de investigación se originó en los Países Bajos, ha tenido impacto a nivel internacional ya que el cuestionario ha sido traducido y validados en más de una docena de idiomas (den Brok et al., 2004; den Brok et al., 2005; den Brok, Tartwijk, Wubbels \& Veldman, 2010; Fraser, Aldridge \& Soerjaningsih, 2010; Fraser $\&$ Walberg, 2005). El cuestionario fue diseñado para su uso en la Educación Secundaria y formó la base de nuevas versiones para maestros de educación primaria (García Bacete, Ferrá Coll, Monjas Casares \& Marande, 2014; Goh \& Fraser, 1996) y profesores de educación superior (Soerjaningsih, Fraser \& Aldridge, 2002). Los investigadores señalan la conveniencia de que este instrumento sea utilizado con mayor fre- 
cuencia por los profesores como una herramienta de retroalimentación para guiar las mejoras en sus relaciones con sus estudiantes en el aula. Se presenta a continuación algunos de los resultados obtenidos en estudios anteriores sobre la confiabilidad del instrumento (ver Tabla 1).

Por lo antes expuesto, el objetivo del presente trabajo fue validar el Cuestionario sobre Relación Docente (QTI) en estudiantes argentinos.

\section{Metodología}

\section{Participantes}

La muestra de carácter intencional estuvo integrada por 256 participantes de ambos sexos (136 varones y 220 mujeres), con edades comprendidas entre los 17 y los 28 años $(M=20.90 ; D E=2.29)$, de nivel socioeconómico medio. Eran alumnos de primero y segundo año de carreras relacionadas con Ciencias Naturales (Geología, Biología, Física y Química) y Ciencias de la Salud (Medicina) de universidades privadas y públicas de la provincia de San Juan (República Argentina).

\section{Instrumento}

El instrumento utilizado es el Cuestionario sobre Relación Docente (QTI) compuesto por 48 ítems en escala Likert de 5 puntos. A partir de las diversas cuestiones planteadas, el estudiante debe seleccionar la opción de respuesta que mejor se ajuste a su situación con respecto a los profesores, en donde 1 corresponde a Muy en desacuerdo, 2 En desacuerdo, 3 Indeciso, 4 De acuerdo y 5 Muy de acuerdo.

Este instrumento permite identificar la tipología de comportamiento predominante en el docente a partir de dos dimensiones: proximidad (cooperación - oposición) e influencia (dominación - sumisión). Dichas categorías son cualidades descriptivas que definen cada dimensión. Asimismo permite analizar los patrones de conducta interpersonal del docente a través de las puntuaciones de sus 8 subdimensiones: líder, estricto, cambiante, insatisfecho, empático, disciplinado, cooperativo y permisivo. A partir de dichas puntuaciones se puede informar a los docentes sus perfiles en el modo de relacionarse con los estudiantes. Los altos puntajes en la dimensión influencia se corresponden con los perfiles: estricto y líder y del mismo modo los perfiles empático, cooperativo corresponden a puntajes elevados en la dimensión proximidad (Wubbels \& Brekelmans, 2005). En la Tabla 2 se presenta la distribución de los ítems y se detallan como ejemplo algunos ítems de cada perfil. Cabe agregar que el instrumento se encabezó con una encuesta sociodemográfica para identificar sexo, edad, institución, carrera, año de la carrera que cursa, de manera de poder caracterizar la muestra estudiada.

\section{Procedimiento}

Se utilizó un muestreo intencional, convocando a los estudiantes de los primeros años de carreras afines a las Ciencias Naturales y Ciencias de la Salud. La recolección de datos fue de carácter voluntario y anónimo, asimismo se contó con el consentimiento informado de los participantes. En el momento de la evaluación se explicó a cada participante el objetivo del estudio. Con el fin de aportar evidencia empírica que avale las cualidades métricas del instrumento seleccionado, se analizó el poder discriminativo de los ítems con la prueba $t$ de Student. Para determinar la validez del constructo se realizó un análisis factorial con el método de Ejes Principales, rotación ortogonal (varimax), cuyo objetivo fue examinar la estructura de la relación entre las variables, permitiendo identificar dimensiones comunes o factores. Por último, se calculó el Alpha de Cronbach para estudiar la fiabilidad. Dichos análisis se realizaron con el software estadístico SPSS.

\section{Resultados}

\section{Análisis de ítems}

Para analizar el poder discriminatorio 
de los 48 ítems del cuestionario se trabajó con el criterio de grupos contrastes. Se analizó, mediante la prueba $t$ de diferencia de medias para muestras independientes, si los sujetos con mayor puntuación (25\% superior del puntaje total) mostraban diferencias significativas en el modo de responder a cada ítem, en comparación con aquellos que obtuvieron un menor puntaje ( $25 \%$ inferior en el puntaje total). Las diferencias de medias obtenidas en todos los ítems, entre los grupos de alto y bajo puntaje en la caracterización de la relación docente, fueron altamente significativas $(p=.000)$ excepto en los ítems 5, 16, 34, 35 y 42 en los que no se hallaron diferencias significativas, razón por la cual fueron descartados para los análisis posteriores.

\section{Estructura factorial. Validez de constructo}

Para estudiar la estructura empírica subyacente del cuestionario, se llevó a cabo un análisis factorial de los 43 ítems restantes, aplicando previamente el índice de adecuación de la muestra de Kaiser-Meyer- Olkin (KMO) que arrojó un coeficiente de .922, lo cual indica que las matrices de los datos son adecuadas para la aplicación del análisis factorial. También se realizó la prueba de esfericidad de Bartlett, que arrojó un valor para chi-cuadrado de $5357.625(p=.000)$, lo que permite rechazar la hipótesis de que la matriz muestral provenga de una población en la que las variables no estén correlacionadas.

Se empleó el método de Componentes Principales sometiéndolo a una rotación varimax dado que esta rotación fue utilizada en estudios anteriores de validación (Fraser et al., 2010). Para la definición de los factores se tuvieron en cuenta las variables que presentaban una carga factorial mayor o igual a .30 y que tuvieran cargas menores a .29 en el resto de los factores. De esta manera se eliminaron los ítems $13,15,38$ y 46 por presentar pesajes complejos y los ítems 20 y 19 en los que se encontró una carga factorial menor a .30. En la nueva factorización volvieron a emerger ítems complejos $(48,47,43$, $24,31)$ que fueron incluidos en el análisis ya que presentan una diferencia de más de .10 puntos entre factores, siendo mayor el pe- saje en el factor que le corresponde. Excepto en el ítem 48, que si bien presenta una carga factorial compleja, desde el punto de vista teórico evalúa un aspecto importante de ese factor (temor) que no es evaluado por otro ítem. Además se ha incluido en el análisis por su aporte tanto en el número de ítems del factor como en la fiabilidad de la escala. En este caso eliminarlo implica modificar sustancialmente el resultado final.

En los análisis realizados se utilizó como punto de corte el método gráfico de Cattell (Gráfico 1), a pesar de que parece mostrar tres factores, el corte se realizó en dos factores debido a que el posible tercer factor explica menos del $6 \%$ de la variancia.

Con dicho método se encontraron dos componentes con autovalores superiores a 2 que explican el $36.62 \%$ de la variancia total, con fuerte peso del Factor 1. Al primer factor le corresponde el $28.51 \%$ de la variancia total explicada, mientras que al Factor 2 le corresponde el $8.11 \%$. Dichos resultados reafirman lo expuesto en la teoría (Wubbels et al., 2012) con respecto a los dos factores que constituyen el cuestionario. En la Tabla 3 se presenta la matriz de los factores ponderados. Como se puede observar los resultados muestran la existencia de dos factores: Factor 1: Proximidad y Factor 2: Influencia. El primer factor bipolar incluye aspectos positivos vinculados a la relación docente (Cooperación) con ítems como: "confían en nosotros" y aspectos negativos (Oposición) con ítems como: "se enojan de forma inesperada". El segundo factor unipolar abarca sólo la dimensión dominación con ítems como: "son estrictos al evaluar trabajos".

\section{Fiabilidad}

Al considerar la fiabilidad de cada factor se obtuvieron los siguientes resultados: para el Factor 1, un Alpha de Cronbach igual a .93 para cooperación y un Alpha de Cronbach igual a .84 para oposición. En el Factor 2, el Alpha es igual a .61.

\section{Discusión y conclusiones}

Ya se ha señalado que la construcción de 
relaciones interpersonales es una condición previa para un clima positivo en el aula. Sin el desarrollo de este aspecto, los docentes no pueden desarrollarse plenamente en su práctica (Wubbels \& Brekelmans, 2005). De allí la importancia de que se preste mayor atención a las habilidades interpersonales en el desarrollo profesional docente, de manera de promover las habilidades comunicativas que les permitan establecer relaciones favorables con los alumnos.

Asimismo los antecedentes manifiestan la necesidad de contar con instrumentos y herramientas de análisis estadísticos que podrían contribuir a desentrañar los vínculos entre las relaciones interpersonales y otras variables (Mainhard et al., 2011). En este sentido, los resultados obtenidos acerca del Cuestionario de Relación Docente permiten afirmar que este instrumento presenta propiedades psicométricas satisfactorias (fiabilidad y validez), ya que se obtuvieron evidencias favorables acerca de la discriminación de los ítems, validez factorial y la confiabilidad del instrumento. Estos resultados son importantes a la hora de comprender la dinámica de los procesos implicados en la relación docente.

Aunque cabe señalar las diferencias en el análisis de la estructura factorial ya que sólo pudo identificarse la categoría dominio como categoría descriptiva de la dimensión influencia. A partir de los resultados obtenidos sólo se pudieron identificar 3 de las 4 categorías que describen las dimensiones propuestas por el modelo teórico que lo sustenta. Es por ello que en este estudio no se han analizado los perfiles que surgen de la combinación de las cuatro categorías. Y si bien el cuestionario original plantea la identificación de ocho perfiles de comportamiento, encontramos estudios que analizan la estructura dual del instrumento (den Brok et al., 2010; García Bacete et al., 2014). El interés de dichas investigaciones, más allá de los perfiles de relación docente, es identificar el grado de proximidad e influencia de los docentes para fomentar conductas que favorezcan el desarrollo de estas dimensiones, las cuales sin duda contribuyen a la mejora de la calidad de la enseñanza.

Asimismo el índice de fiabilidad es menor en comparación con los puntajes obtenidos en la versión original. Consideramos que esto puede atribuirse a las características del contexto universitario en donde el docente asume más bien un papel protagónico en la formación profesional de sus estudiantes y difícilmente encarna un rol sumiso en la relación docente-alumno. En este sentido sería conveniente una revisión de los ítems correspondientes a dicha categoría, como así también la necesidad de ampliar los ítems que corresponden a la categoría dominio para obtener mejores índices de confiabilidad. Los aspectos señalados deberían ser tenidos en cuenta en futuras investigaciones. Otros de los retos de próximos estudios será seguir analizando el efecto de la interacción entre las dimensiones interpersonales (influencia y proximidad), cuestión central de la teoría interpersonal.

Los antecedentes señalan la conveniencia de que este instrumento sea utilizado con mayor frecuencia por los profesores como una herramienta de retroalimentación para guiar las mejoras en sus relaciones con sus estudiantes en el aula. Teniendo en cuenta los resultados obtenidos se considera que el Cuestionario de Relación Docente puede ser usado como guía para mejorar las relaciones interpersonales y ayudar al profesorado en su desarrollo profesional lo cual puede considerarse una aportación positiva de este estudio. La utilización de este instrumento puede ser una herramienta valiosa, tanto para actividades de investigación como en programas de prevención.

Los buenos docentes deben ser capaces de empatizar con los alumnos, escuchar mejor y comprender su mundo. En este sentido se requiere de planes formativos para que el profesorado tome conciencia de la dimensión relacional de su práctica educativa y reciba la formación necesaria para poder optimizar el gran potencial educativo que esta tiene en el desarrollo de sus estudiantes. En efecto, el integrar en la capacitación del docente estrategias para desarrollar y fortalecer las competencias relacionales favorecerá: en el alumnado, relaciones interpersonales edificantes que contribuirá en el rendimiento académico y, en el 
中ạ̧⿻日乚冖e, facilitará su desempeño y éxito pro-

ferjpaale Cronbach obtenidos en estudios anteriores

\begin{tabular}{llccc}
\hline & Perfiles & $\begin{array}{c}\text { Den Brok } \\
(2005)\end{array}$ & $\begin{array}{c}\text { Den Brok } \\
(2010)\end{array}$ & $\begin{array}{c}\text { Fraser } \\
(2010)\end{array}$ \\
\hline DC & Lider & .85 & .88 & .83 \\
\hline $\mathrm{CD}$ & Cooperativo & .75 & .84 & .78 \\
\hline $\mathrm{CS}$ & Empático & .84 & .86 & .77 \\
\hline $\mathrm{SC}$ & Permisivo & .70 & .62 & .80 \\
\hline $\mathrm{SO}$ & Cambiante & .86 & .76 & .83 \\
\hline $\mathrm{OS}$ & Insatisfecho & .89 & .78 & .87 \\
\hline OD & Disciplinado & .84 & .81 & .85 \\
\hline DO & Estricto & .79 & .69 & .65 \\
\hline
\end{tabular}

Nota: D: Dominio, C: Cooperación, S: Sumisión, O: Oposición

Tabla 2

Descripción de los Perfiles Cuestionario sobre la Relación Docente (QTI)

\begin{tabular}{clcl}
\hline Categoría & Perfiles & $\begin{array}{c}\text { N de } \\
\text { items }\end{array}$ & \multicolumn{1}{c}{ Ejemplo de items } \\
\hline DC & Lider & 8 & $\begin{array}{l}\text { "Explica la materia con claridad", "Actúa con } \\
\text { confianza" }\end{array}$ \\
\hline CD & Cooperativo & 8 & $\begin{array}{l}\text { "Es amable", "Las clase con este profesor son } \\
\text { agradables" }\end{array}$ \\
\hline CS & Empático & 8 & $\begin{array}{l}\text { "Se da cuenta cuando no entendemos", "Tiene } \\
\text { paciencia" }\end{array}$ \\
\hline SC & Permisivo & 8 & $\begin{array}{l}\text { "Nos hace perder el tiempo en clase", "Nos } \\
\text { permite salir mucho de clase". }\end{array}$ \\
\hline SO & Cambiante & 8 & $\begin{array}{l}\text { "Es vacilante", "Actúa como si no supiera qué } \\
\text { hacer" }\end{array}$ \\
\hline OS & Insatisfecho & 8 & $\begin{array}{l}\text { "Piensa que no sabemos nada", "Piensa que no } \\
\text { podemos hacer las cosas bien" }\end{array}$ \\
\hline OD & Disciplinado & 8 & $\begin{array}{l}\text { "Se enoja rápidamente", "Es impaciente". } \\
\text { DO }\end{array}$ Estricto \\
\hline
\end{tabular}

Nota: D: Dominio, C: Cooperación, S: Sumisión, O: Oposición 
Tabla 3

Análisis Factorial, método de extracción componentes principales, solución varimax correspondiente a los items del Cuestionario sobre la Relación Docente (QTI)

\begin{tabular}{|c|c|c|}
\hline & \multicolumn{2}{|c|}{ Componente } \\
\hline & 1 & 2 \\
\hline 29 Son amables (Cooperación) & .791 & -.054 \\
\hline 45 Las clases de estos profesores son agradables (Cooperación) & .759 & -.055 \\
\hline 10 Están dispuestos a explicar las cosas de nuevo (Cooperación) & .753 & -.011 \\
\hline 25 Nos ayudan con nuestro trabajo (Cooperación) & .751 & -.088 \\
\hline 30 Podemos influir sobre estos profesores (Cooperación) & .750 & -.031 \\
\hline 22 Son pacientes (Cooperación) & .723 & -.124 \\
\hline 33 Puedo apoyarme en este profesor (Cooperación) & .685 & -.023 \\
\hline $14 \mathrm{Si}$ tenemos algo que decir, estos profesores pueden escucharnos (Cooperación) & .665 & .084 \\
\hline 2 Confían en nosotros (Cooperación) & .643 & -.119 \\
\hline 9 Captan nuestra atención (Cooperación) & .631 & .114 \\
\hline 21 Actúan con confianza (Cooperación) & .596 & .137 \\
\hline 26 Podemos decidir algunas cosas en clase con estos profesores (Cooperación) & .596 & -.058 \\
\hline 41 Aceptan una broma (Cooperación) & .588 & .009 \\
\hline 1 Hablan con entusiasmo sobre su materia (Cooperación) & .566 & .113 \\
\hline 37 Tienen sentido del humor (Cooperación) & .546 & .032 \\
\hline 18 Se dan cuenta cuando no entendemos (Cooperación) & .508 & .141 \\
\hline $6 \mathrm{Si}$ no estamos de acuerdo con estos profesores, podemos hablar de ello (Cooperación) & .467 & -.007 \\
\hline 23 Es fácil ridiculizar a estos docentes (Oposición) & -.373 & -.052 \\
\hline 7 Son vacilantes (Oposición) & -.380 & .233 \\
\hline 27 Piensan que hacemos trampa (Oposición) & -.406 & .285 \\
\hline 31 Piensan que no sabemos nada (Oposición) & -.495 & .345 \\
\hline 24 Son sarcásticos (Oposición) & -.496 & .373 \\
\hline 11 Actúan como si no supieran qué hacer (Oposición) & -.497 & .104 \\
\hline $8 \mathrm{Se}$ enojan rápidamente (Oposición) & -.530 & .196 \\
\hline 4 Se enojan de forma inesperada (Oposición) & -.532 & .218 \\
\hline 43 Parecen insatisfechos (Oposición) & -.590 & .393 \\
\hline 47 Son desconfiados (Oposición) & -.598 & .404 \\
\hline 39 Piensan que no podemos hacer las cosas bien (Oposición) & -.651 & .262 \\
\hline 40 Son muy exigentes (Dominio) & .042 & .634 \\
\hline 36 Los exámenes de estos profesores son difíciles (Dominio) & -.194 & .551 \\
\hline 28 Son estrictos (Dominio) & .154 & .522 \\
\hline 44 Son estrictos al evaluar trabajos (Dominio) & .155 & .511 \\
\hline 48 Tenemos miedo de estos profesores (Dominio) & -.433 & .461 \\
\hline 17 Son líderes (Dominio) & .074 & .394 \\
\hline 32 Tenemos que estar en silencio en la clase de estos profesores (Dominio) & .022 & .380 \\
\hline 12 Son demasiado rápidos para corregir cuando rompemos una regla (Dominio) &,- .071 & .364 \\
\hline 3 Parecen inseguros (Dominio) & -.298 & .343 \\
\hline Porcentaje de Varianza por factor: & $28.51 \%$ & $8.11 \%$ \\
\hline Porcentaje de Varianza Total: & 36 & \\
\hline
\end{tabular}




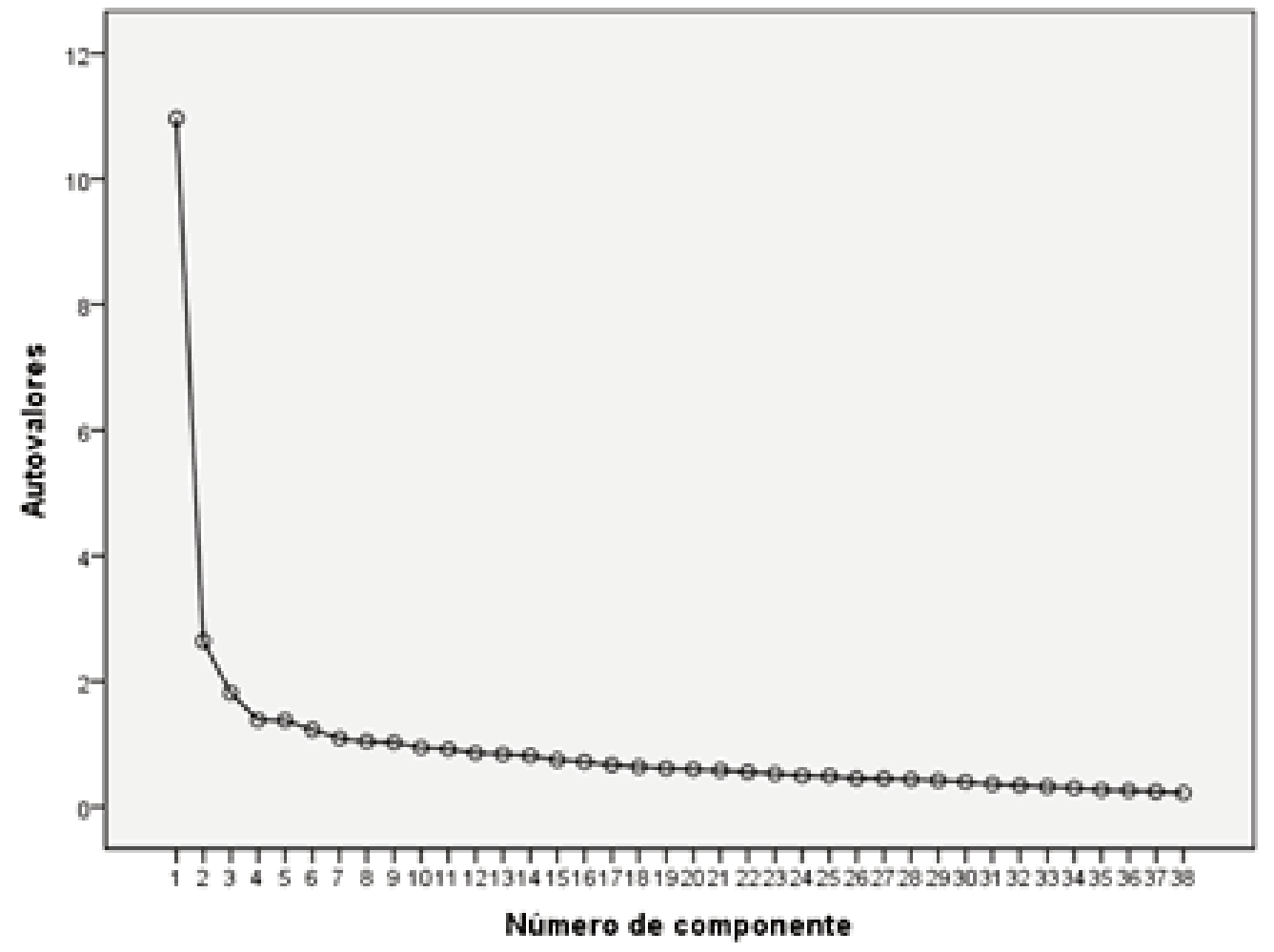

Grafico 1

Gráfico de Sedimentación 


\section{Referencias bibliográficas}

Abarca, M., Marzo, L., \& Sala, J. (2002). La educación emocional y la interacción profesor/aalumno/a [Emotional education and teacherstudent interaction]. Revista Electrónica Interuniversitaria de Formación del Profesorado, 5, 1-4. Recuperado el 4 de marzo de 2014 de http://www.aufop.org/publica/reifp/02v5n3. asp

Aldridge, J., Laugksch, R. C., Seopa, M. A., \& Fraser, B (2006). Development and validation of an instrument to monitor the implementation of outcomes-based learning environments in science classrooms in South Africa. International Journal of Science Education, 28(1), 45-70. http://dx.doi.org/10.1080/09500 690500239987

Bibi, F., Kazmi, S.F., Chaudhry, A.G., \& Khan, S.E. (2015). Relationship Between Emotional Intelligence and Coping Strategies Among University Teachers of Khyber Pakhtunkhwa. Pakistan Journal of Science, 67, 81-84 Recuperado el 4 de marzo de 2014 de https://www.researchgate.net/publication/277668053_RELATIONSHIP_BETWEEN EMOTIONAL INTELLIGENCE A ND_COPING_STRATEGGIES_AMONG_ŪNI VERSITY_TEACHERS_OF_KHYBER_PAK HTUNKHWA.

Brackett, M., Palomera, R., Mojsa-Kaja, J., Reyes, M.R. \& Salovey, P. (2010). EmotionRegulation Ability, Burnout, and Job Satisfaction among British Secondary-School Teachers. Psychology in the Schools, 47(4). http://dx.doi.org/10.1002/pits

Carbonero, M., Martín-Antón, L. \& Reoyo, N. (2011). El profesor estratégico como favorecedor del clima de aula [The strategic teacher as conducive to classroom climate]. European Journal of Education and Psychology, 4, 133142. Recuperado el 10 de abril de 2014 de http://www.redalyc.org/articulo.oa?id=1293226 59004.

Cohen, J. H. \& Amidon, E. J. (2004). Reward and punishment histories: A way of predicting teaching style? Journal of Educational Research, 97(5). http://dx.doi.org/10.3200/JOER.97.5.
269-280

Coll, C. \& Sánchez, E. (2008). Presentación. El análisis de la interacción alumno-profesor: líneas de investigación [Presentation. The analysis of the pupil-teacher interaction: Researching lines]. Revista de Educación, 346, 15-32 Recuperado el 10 de abril de 2014 de http:// www.redalyc.org/pdf/280/28041012002.pdf.

Choi, K., Vickers, K. \& Tassone, A. (2014). Trait emotional intelligence, anxiety sensitivity, and experiential avoidance in stress reactivity and their improvement through psychological methods. Europe's Journal of Psychology, 10(2), 376-404.http://dx.doi.org/10.5964/ejop.v10i2. 754

Darling-Hammond, L. (2008). Teacher quality definition debates: What is an effective teacher? En S. Publications (Ed.), 21st Century Education: A Reference Handbook. University of Arizona, Tuscon: Sage Publications.

Delgado Arriagada, T. (2004). La disposición emocional en el aula universitaria y su relación con la calidad educativa en la formación inicial docente [The relationship between emotional aptitude in the universitary classroom and the inicial teacher's training quality]. Revista Electrónica de Diálogos Educativos, 4, 17 - 23. Recuperado el 10 de abril de 2014 de http://132. 248.9.34/hevila/Revistaelectronicadialogos educativos/2004/ vol4/no7/3.pdf.pdf.

den Brok, P., Fisher, D., \& Scott, R. (2005). The importance of teacher interpersonal behaviour for student attitudes in Brunei primary science classes. International Journal of Science Education, 27(7), 765-779. http://dx.doi.org/ 10.1080/09500690500038488

den Brok, P., Brekelmans, M., \& Wubbels, T. (2004). Interpersonal teacher behaviour and student outcomes. School Effectiveness \& School Improvement, 15(3/4), 407-442. http://dx.doi.org $/ 10.1080 / 09243450512331383262$

den Brok, P., Brekelmans, M. \& Wubbels, T. (2006). Multilevel issues in research using students' perceptions of learning environments: The case of the questionnaire on teacher interaction. Learning Environments Research, 9(3), 199-213. http://dx.doi.org/10.1007/s1 0984-006-9013-9

den Brok, P., Tartwijk, J., Wubbels, T., \& Veldman, L. (2010). The differential effect ofthe teacher- 
student interpersonal relationship on student outcomes for students with different ethnic backgrounds. Brittsh Journal of Educational Psychology, 80, 199-221.

Di Fabio, A. (2015). Beyond fluid intelligence and personality traits in social support: The role of ability based emotional intelligence. Frontiers in Psychology, 6, 1-6. http://dx.doi.org/ 10.3389/ fpsyg.2015.00395

Fernández-Abascal, E., \& Martín-Díaz, M. (2015). Dimensions of emotional intelligence related to physical and mental health and to health behaviors. Frontiers in Psychology, 6. http://dx.doi. org/10.3389/fpsyg.2015.00317

Fernández Berrocal, P. \& Ruiz Aranda, D. (2008). La inteligencia emocional en la educación. [Emotional intelligence in education]. Revista Electrónica de Investigación Psicoeducativa, 6, 421-436. Recuperado el 10 de marzo de 2014 de http://www. investigacion-psicopedagogica.org/revista/articulos/15/espannol /Art_15_256.pdf.

Fraser, B., Aldridge, J. \& Soerjaningsih, W. (2010). Instructor-Student Interpersonal Interaction and Student Outcomes at the University Level in Indonesia. Open Education Journal, 3, 21-33. Recuperado el 10 de marzo de 2014 de https://benthamopen.com/contents/pdf/TOEDUJ/TOEDUJ-3-21.pdf.

Fraser, B. \& Walberg, H.J. (2005). Research on teacher-student relationships and learning environments: Context, retrospect and prospect. International Journal of Educational Research, 43(1/2), 103-109. http://dx. doi.org/ 10.1016/j.ijer.2006.03.001

García Bacete, F., Ferrá Coll, P., Monjas Casares, M.I. \& Marande, G. (2014). Las relaciones del profesorado con el alumnado en aulas del ciclo inicial de Educación Primaria. Adaptación del Questionnaire on Teacher Interaction-Early Primary (QTI-EP) [The relationship between teachers and students in inicial course of primary education classrooms]. Revista de Psicodidáctica, 19(1), 211-231. http://dx.doi. org/10.1387/RevPsicodidact.9081

Gargallo López, B. (2008). Estilos de docencia y evaluación de los profesores universitarios y su influencia sobre los modos de aprender de sus estudiantes [Teaching styles and evaluation of university professors and their influence on the learning of their students]. Revista Española de Pedagogía, 425-445. Recuperado el 10 de mayo de 2014 de https://dialnet.unirioja. es/descarga/articulo/2709000.pdf.

Gargallo López, B., Sánchez Peris, F.J., Ros, C. \& Ferreras Remesal, A. (2010). Estilos docentes de los profesores universitarios: La percepción de los alumnos de los buenos profesores [Teaching styles of university professors: The perception of the students of good teachers]. Revista Iberoamericana de Educación, 51. Recuperado el 10 de mayo de 2014 de www.rieoei.org/delos lectores/ 3236 Lopez. pdf.

Goh, S.C. \& Fraser, B.J. (1996). Validation of an elementary school version of the questionnaire on teacher interaction. Psychological Reports, 79(2). http://dx.doi.org/10.2466/pr0.1996.79.2.515

Holinka, C. (2015). Stress, emotional intelligence, and life satisfaction in college students. College Student Journal, 49, 300-311. Recuperado el 10 de octubre de 2015 de http://eric.ed. gov/?id= EJ1095697.

Ivcevic, Z. \& Brackett, M. (2014). Predicting school success: Comparing Conscientiousness, Grit, and Emotion Regulation Ability. Journal of Research in Personality, 52, 29-36. http://dx.doi.org/10.1016/j.jrp.2014.06.005

Jong, R., Mainhard, T., Tartwijk, J., Veldman, I., Verloop, N. \& Wubbels, T. (2014). How preservice teachers' personality traits, self-efficacy, and discipline strategies contribute to the teacher-student relationship. British Journal of Educational Psychology, 84(2), 294-310. http://dx.doi.org/10.1111/bjep.12025

Khine, M.S. \& Fisher, D.L. (2004). Teacher interaction in psychosocial learning environments: Cultural differences and their implications in science instruction. Research in Science \& Technological Education, 22(1), 99-111. http://dx.doi.org/10. 1080/026351404200 018 7566.

Klein, F. (2011). Las tensiones en la relación docente-alumno. Una investigación del ámbito 
educativo [The stress in teacher-student relationship. An investigation of educational field]. Aposta Revista de Ciencias Sociales, 51, 1-28. Recuperado el 10 de marzo de 2014 de http:// www.apostadigital. com/revistav3/hemeroteca/ fklein3.pdf.

LaBillois, J.M. \& Lagacé-Séguin, D.G. (2009). Does a good fit matter? Exploring teaching styles, emotion regulation, and child anxiety in the classroom. Early Child Development \& Care, 179(3), 303-315. http://dx.doi.org/ 10.1080/03004430601078636

Lapointe, J.M., Legault, F. \& Batiste, S. J. (2005). Teacher interpersonal behavior and adolescents' motivation in mathematics: A comparison of learning disabled, average, and talented students. International Journal of Educational Research, 43(1/2), 39-54. http://dx.doi.org/ 10.1016/j.ijer. 2006.03.005

Látalová, V. \& Pilárik, L. (2015). Predicting career decision-making strategies in women: The role of self-determination and perceived emotional intelligence. Studia Psychologica, 57(2), 95114.

Limonero, J.T., Fernández-Castro, J., Soler-Oritja, J., Álvarez-Moleiro, M., Fernández-Abascal, E. G. \& Tousignant, M. (2015). Emotional intelligence and recovering from induced negative emotional state. Frontiers in Psychology, June, 1-8. http://dx.doi.org/10.10.3389/fpsyg. 2015.00816

Mainhard, M.T, Brekelmans, M. \& Wubbels, T. (2011). Coercive and supportive teacher behaviour: Within- and across-lesson associations with the classroom social climate. Learning \& Instruction, 21(3), 345-354. http://dx.doi.org /10.1016/j.learninstruc.2010.03.003

Mikulic, I.M., Crespi, M.C. \& Radusky, P. (2015). Construcción y validación del Inventario de Competencias Socioemocionales para adultos (ICSE) [Designing and validation of Adult Socioemocional Competences Inventory (SECI)]. Interdisciplinaria, 32(1), 1-17. Recuperado el 10 de noviembre de 2016 de www.redalyc.org/pdf/180/18043528007.pdf.
Moreno García, R. (2010). Estilos de apego en el profesorado y percepción de sus relaciones con el alumnado [Attachment styles in teacher and perception of their relationships with students]. Tesis Doctoral no publicada. Universidad Complutense de Madrid. Madrid.

Mortan, R.A., Ripoll, P., Carvalho, C. \& Bernal, M.C. (2014). Effects of emotional intelligence on entrepreneurial intention and self-efficacy. Journal of Work and Organizational Psychology, 30(3), 97-104. http://dx.doi.org /10.1016/j.rpto.2014.11.004

Nijveldt, M., Beijaard, D., Brekelmans, M., Verloop, N. \& Wubbels, T. (2005). Assessing the interpersonal competence of beginning teachers: The quality of the judgement process. International Journal of Educational Research, 43(1/2), 89-102. http://dx.doi.org/10.1016/j. ijer.2006.03.008

Oros, L. \& Fontana Nalesso, A. (2015). Niños socialmente hábiles: ¿Cuánto influyen la empatía y las emociones positivas? [Socially skillful children: how much influence do positive emotions and empathy have?]. Interdisciplinaria, 32(1), 109-125. Recuperado el 15 de noviembre de 2015 de http://www.redalyc.org/pdf/180 /18041090006.pdf.

Paes da Silva, M. J. \& Kalil de Freitas Castro, R. (2003). Influencia del comportamiento comunicativo verbal y no verbal del docente en el aula. Visión de los alumnos y docentes de Enfermería [Teacher's verbal and no verbal sociable behaviour influence in the classroom. Students and nursing's teachers view]. Enfermería Global - Universidad de Murcia, 3 1-11 Recuperado el 10 de mayo de 2012 de https://digitum.um.es/xmlui/bitstream/ 10201/23940/2/621-2867-2-PB\%5B1 $\% 5$ D.pdf.

Páez Cala, M. L. \& Castaño Castrillón, J. J. (2015). Inteligencia Emocional y Rendimiento Académico en Estudiantes Universitarios. [Emotional Intelligence and academic performance in university students]. Psicología desde el Caribe, 32, 268-285. Recuperado el $10 \mathrm{de}$ mayo de 2012 de http://www.redalyc.org/pdf/ 213/213 41030006.pdf. 
Palomera M.R, Gil-Olarte Márquez, P. \& Brackett, M. (2006). ¿Se perciben con inteligencia emocional los docentes? Posibles consecuencias sobre la calidad educativa. [Are teachers noticed with Emotional Intelligence? Possible consequences in educational quality]. Revista de Educación, 341, 687-703. Recuperado el 10 de mayo de 2012 de https://www.researchgate.net/publication/28132778_Se_perciben_con_inteligencia_emocional_los_docentes_posibles_consecue ncias_sobre la calidad educativa.

Palomera Martín, R., Fernández-Berrocal, P. \& Brackett, M. (2008). La inteligencia emocional como una competencia básica en la formación inicial de los docentes: Algunas evidencias. Revista Electrónica de Investigación Psicoeducativa, 6(2), 437-454.

Palomera, R., Fernández-Berrocal, P. \& Brackett, M. A. (2008). La inteligencia emocional como una competencia básica en la formación inicial de los docentes: Algunas evidencias [Emotional Intelligence as an elementary competence in the initial training of teachers: some evidences]. Revista Electrónica de Investigación Psicoeducativa, 6(15), 437-454. Recuperado el 10 de mayo de 2012 de http://www.investigacion-psicopedagogica.org/revista/ articulos/15/espannol/Art 15 276.pdf.

Páramo, P. (2008). Factores psicosociales asociados a la evaluación del docente [The Psychosocial Factors Associated with Teacher Assessment]. Educación y Educadores, 11-30. Recuperado el 10 de mayo de 2012 de http://educacionyeducadores.unisabana. edu.co/index.php/eye/article/view/715/1693.

Pena, M. \& Extremera, N. (2012). Inteligencia emocional percibida en el profesorado de Primaria y su relación con los niveles de burnout e ilusión por el trabajo (engagement). Revista de Educación, 359 (Septiembre-diciembre ), 604-627. http://dx.doi.org.10.4438/ 1988-592X-RE-2011-359-109

Rayneri, L.J., Gerber, B.L. \& Wiley, L.P. (2006). The relationship between classroom environment and the learning style preferences of gifted middle school students and the impact on levels of performance. Gifted Child Quarterly, 50(2), 104-118. http://dx.doi.org/10.1177/00169 8620605000203

Rocha Chávez, R. (2013). Escala de Opinión de los estudiantes sobre la Efectividad de la Docencia en Educación Superior [Scale of the Student Opinion about Teacher Effectiveness (SOTES) in Higher Education]. Formación Universitaria, 6(6), 13-22. http://dx.doi.org/10.4067/S071850062013000600003

Saft, E.W. \& Pianta, R.C. (2001). Teachers' perceptions of their relationships with students: Effects of child age, gender, and ethnicity of teachers and children. School Psychology Quarterly, 16(2), 125-141. http://dx.doi.10.1521 /scpq.16.2.125.18698

Salazar, S. (2006). Hacia una caracterización del docente universitario "excelente": Una revisión a los aportes de la investigación sobre el desempeño del docente universitario. [Towards a characterization of the "excellent" university teacher: A review of the contributions of research on university teacher performance]. Revista Educación y Pedagogía, 30, 31-49. Recuperado el 10 de mayo de 2012 de www.redalyc.org/articulo.oa?id=44030103.

Samper-García, P., Mesurado, B., Richaud, C. \& Llorca, A. (2016). Validación del cuestionario de conciencia emocional en adolescentes españoles [Validation of emotional awareness Questionnaire in spanish adolescents]. Interdisciplinaria, 33(1), 1-14.

She, H.C. \& Fisher, D. (2000). The Development of a Questionnaire to Describe Science Teacher Communication Behavior in Taiwan Science Classrooms. Science Education, 84(6).

Soerjaningsih, W., Fraser, B. \& Aldridge, J. (2002). Instructor-student interpersonal behavior and student outcomes at the university level in Indonesia. Paper presented at the Annaul Mettining of the American Educational Research Association, New Orleans.

Telli, S., den Brok, P. \& Cakiroglu, J. (2010). The importance of teacher-student interpersonal relationships for Turkish students' attitudes towards science. Research in Science \& 
Technological Education, 28(3), 261-276. $\mathrm{http} / / / \mathrm{dx}$.doi.org/10.1007/s10984-007-9023-2

Vaillant, D. (2005). Formación de Docentes en América Latina. Re-inventando el modelo tradicional [Teacher training in Latin America. Reinventing the traditional model]. Barcelona: Octaedro.

Vaillant, D. (2007). Mejorando la formación y el desarrollo profesional docente en América Latina [Improving teacher training and professional development in Latin America]. Revista Pensamiento Educativo, 41. Recuperado el 10 de mayo de 2012 de http://www.ub.edu/obipd/PDF\%20docs/Form aci $\%$ C3\%B3\%20Permanent/Educaci $\% C 3 \% B$ 3\%20Primaria/Publicacions/Mejorando\%201a $\% 20$ formaci $\%$ C3\%B3n $\% 20 y \% 20$ el $\% 20$ desarrollo\%20profesional\%20docente $\% 20$ en $\% 2$ 0Latinoam\%C3\%A9rica.\%20Vaillant,D.pdf.

Velasco, J. \& Alonso de González, L. (2008). Sobre la teoría de la educación dialógica [About the theory of dialogical education]. EDUCERE, 12, 461-470. Recuperado el 10 de mayo de 2012 de www.redalyc.org/pdf/356/35614569006.pdf.

Veldman, I., van Tartwijk, J., Brekelmans, M. \& Wubbels, T. (2013). Job satisfaction and teacher-student relationships across the teaching career: Four case studies. Teaching \& Teacher Education, 32, 55-65. http://dx.doi.org/10.1016/ j.tate.2013.01.005

Vidal, L. (2002). Evaluación organizacional de la excelencia docente [Organizational evaluation of teaching excellence]. Magíster en Administración Educacional. Universidad de Playa Ancha de Valparaíso, Chile.

Villa Sánchez, A. (2008). La excelencia docente [Teaching excellence]. Revista de Educación, número extraordinario, 177-212. Recuperado el 10 de mayo de 2012 de www.revistaeducacion.mec.es/re2008/re2008_08.pdf.
Wentzel, K. (1997). Student motivation in middle school: the role of perceived pedagogical caring. Journal of Educational Psychology in the Schools, 89(3), 411-419. http://dx.doi.org/ 10.1037/0022-0663.89.3.411

Wubbels, T., Brekelmans, M., \& Hooymayers, H. (1991). Interpersonal teacher behavior in the classroom. En B.J. Fraser \& H.J. Walberg (Eds.), Educational environments: Evaluation, antecedents and consequences (pp. 141-160). Oxford, England: Pergamon Press.

Wubbels, T. \& Brekelmans, M. (2005). Two decades of research on teacher-student relationships in class. International Journal of Educational Research, 43(1/2), 6-24. http://dx.doi.org/ 10. 1016/j.ijer.2006.03.003

Wubbels, T., den Brok, P., van Tartwijk, J. \& Levy, Jack (Eds.). (2012). Interpersonal Relationships in Education An Overview of Contemporary Research (Vol. 3). The Netherlands: Sense Publishers.

Wubbels, T., \& Levy, J. (1997). Paying attention to relationships. Educational Leadership, 54(7).

Zabalza, M. A. (2003). Competencias docentes del profesorado universitario. Calidad y desarrollo profesional. [Teaching competencies of university teacher. Quality and professional development]. Madrid: Narcea.

Zapata, J., Rojas, L., \& Gómez, A. (2010). Modelado de la relación de confianza profesor-estudiante en la docencia universitaria [Modeling teacher-student confidence in college teaching]. Educación y Educadores, 13. Recuperado el 10 de mayo de 2012 de www.redalyc.org/pdf/834/83416264006.pdf.

Zhang, L.F. (2011). Teaching styles and conceptions of effective teachers: Tibetan and Han Chinese academics compared. Learning \& Individual Differences, 21(5), 619-623. http:// dx.doi.org/10.1016/j.lindif.2011.06.005

Fecha de recepción: 29 de agosto de 2016 Fecha de aceptación: 4 de septiembre de 2017 